This item was submitted to Loughborough's Research Repository by the author.

Items in Figshare are protected by copyright, with all rights reserved, unless otherwise indicated.

\title{
Communication-aware convoy following guidance for UAVs in a complex urban environment
}

PLEASE CITE THE PUBLISHED VERSION

http://dx.doi.org/10.1109/MED.2016.7535884

PUBLISHER

(C) IEEE

VERSION

AM (Accepted Manuscript)

LICENCE

CC BY-NC-ND 4.0

\section{REPOSITORY RECORD}

Oh, Hyondong, Hyo-Sang Shin, Seungkeun Kim, Pawel Ladosz, and Wen-Hua Chen. 2016. "Communicationaware Convoy Following Guidance for Uavs in a Complex Urban Environment". figshare.

https://hdl.handle.net/2134/22720. 


\title{
Communication-Aware Convoy Following Guidance for UAVs in a Complex Urban Environment
}

\author{
Hyondong Oh, Hyo-Sang Shin, Seungkeun Kim, Pawel Ladosz, and Wen-Hua Chen
}

\begin{abstract}
This paper proposes a communication-aware trajectory planning approach for UAVs to relay data/information (e.g. live surveillance feed) between a ground control station and friendly ground vehicles (a convoy) moving in a complex urban area. UAVs are controlled to stay: $i$ ) within the communicationfeasible area (having a direct line-of-sight to the moving convoy and within the maximum communication range) and ii) as close as possible to the convoy to have better communication quality, while satisfying their kinematic and dynamic constraints. Numerical simulations and a proof-of-concept indoor flight test have been performed to validate the benefit and feasibility of the proposed algorithm.
\end{abstract}

\section{INTRODUCTION}

Maintaining communication amongst mobile agents in a networked team is quite challenging due to limited bandwidth, maximum communication range, transmission power, and physical obscuration or occlusion in the mission environment. An unmanned aerial vehicle (UAV) can be used as communication relay to allow a ground operator/system to have sufficient datalink to effectively see beyond the communication range and over the horizon/buildings where traditional methods fail. The relay UAV can also be used to transmit/share critical information efficiently from an operator to an end-user or between vehicles. Exploiting UAVs for communication relay would be beneficial over ground vehicles in the sense that: i) there is less signal attenuation and interference in ground-to-air communication and ii) UAVs can be rapidly deployed without being confined to the prescribed roadmap.

For effective trajectory planning of the relay UAV considering communication constraints, it is essential to predict the communication performance in order to assess the feasibility of the trajectories. To make these predictions, a certain model or measured metric of the communication environment is required, which can be largely categorised into [1]: i) modelbased approaches where a model of the communication environment is used to predict the communication performance of the networked team and ii) measurement-based approaches

This work was supported by the UK Engineering and Physical Science Research Council (EPSRC) under the Grant EP/J011525/1.

Hyondong Oh, Pawel Ladosz and Wen-Hua Chen are with the Department of Aeronautical and Automotive Engineering, Loughborough University, Loughborough, Leicestershire, LE11 3TU, United Kingdom \{h.oh, p.ladosz, w.chen\}elboro.ac.uk

Hyo-Sang Shin is with the Institute of Aerospace Sciences, Cranfield University, Cranfield, Bedfordshire, MK43 OAL, United Kingdom h.shin@cranfield.ac.uk

Seungkeun Kim is with the Department of Aerospace Engineering, Chungnam National University, 99 Daehak-ro, Yuseong-gu, Daejeon 305764, Republic of Korea skim78@ cnu.ac.kr which make use of online communication quality measurements. Model-based approaches can be further categorised into: i) range-only, ii) range and visibility, and iii) channel propagation. Most model-based ones considered range-based communication constraints [2]-[5], while only few of them considered visibility [6] and a channel propagation model [7]-[9]. For measurement-based approaches, the signal-tonoise ratio (SNR) or similar channel metrics are measured, and a gradient following method [10], [11] is used to guide the vehicle gradually towards the area which produces higher SNR, without relying on the pre-specified communication model and location information.

It is worthwhile noting that most of existing UAV communication relay approaches employ a single multi-rotor UAV (e.g. helicopter or quadrotor which can hover) and a simple distance-based communication model. These might be vulnerable to the failure of the relay UAV or inefficient to an uncertain and dynamic environment. If ground mobile nodes move dynamically, then an optimal networking structure and the corresponding desired relay UAV position to efficiently share/transmit data between them might change significantly, which might be difficult to be followed by the multi-rotor UAV due to its limited mobility. Besides, many of them consider a 2-D (two dimensional) open field environment where there are no buildings or obstacles, which is unrealistic.

To address this issue, this paper proposes a 3-D communication-aware trajectory planning approach to guide multiple fixed-wing (rather than multi-rotor) UAVs continuously to relay information (e.g. live surveillance feed) between the ground control station (GCS) and friendly ground vehicles (termed as a convoy hereafter) moving in an urban area. Maintaining a direct line-of-sight (LOS) between ground mobile units is important to ensure desired communication quality and robustness. However, in urban environments, this is quite challenging due to a large number of differently shaped buildings and obstacles [12]. Depending on the buildings, communication loss might vary significantly; thus it is required to avoid this situation (i.e. losing the direct LOS). Therefore, this study particularly focuses on dealing with communication constraints (limited communication capability and LOS block by buildings) for relay UAV trajectory planning while satisfying kinematic constraints (speed and turning rates) of fixed-wing UAVs.

The structure of this paper is given as: Section 2 presents the assumptions and overview of the proposed algorithm. Section 3 describes communication feasible area and desired loitering orbit generation, followed by the convoy following trajectory planning algorithm based on the Lyapunov vector 
field guidance in Section 4. Section 5 presents numerical simulation results and a proof-of-concept flight test. Lastly, conclusions and a future work are discussed in Section 6.

\section{Trajectory Planning Approach OVERVIEW}

There are several assumptions made in the study. First, the urban environment is assumed to be given including the number of buildings and their location and dimension. Vehicles in a convoy are assumed to be able to communicate each other as they are in the close proximity, so they are regarded as one convoy unit/vehicle. Trajectory of the convoy for a certain mission is known (or can be estimated) to the GCS. A convoy is assumed to move on a 2-D plane (i.e. altitude is zero). Relay UAVs share a known global coordinate system for their own and convoy position. A communication between nodes (relay UAVs, a convoy and a GCS) is modelled by the communication range and visibility, so if they are within a certain distance and there exists a direct LOS (not obstructed by buildings) between them, it is assumed that they can communicate with each other.

Figure 1 shows the flow chart of the proposed approach on communication-aware convoy following trajectory planning. First, a 3-D communication-feasible area is obtained for the current convoy location in the region of interest (ROI). Then, to make the fixed-wing UAV fly within this area while being as close as possible to the ground convoy, the appropriate desired loitering orbit is determined for the UAV to follow. Note that this communication-feasible area and the orbit could be generated in real time for each convoy position at a fixed time rate. However, if the computation capacity is limited, this process can be made offline by computing the orbits for a sufficient number of representative points in the ROI and use them as training data set for the Gaussian Process regression (GPR). Then, the desired orbit for the online/current convoy position can be rapidly predicted by the GPR in real time. To make the UAV follow the desired loitering orbit at the desired height, a Lyapunov vector field guidance (LVFG) approach is used. From the next section, more detailed explanation on each element is followed.

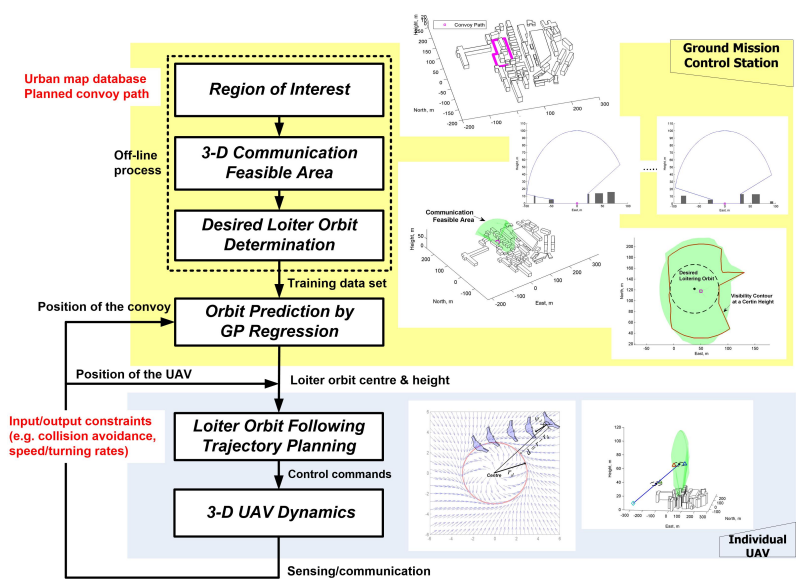

Fig. 1. Flow chart of the proposed communication-aware convoy following trajectory planning process.

\section{Communication-FeAsible Area And Orbit GENERATION}

\section{A. 3-D Visibility region with communication range}

An urban environment space can be mathematically defined as:

$$
\mathbf{X}_{E}=\left\{(x, y, z) \in \mathbb{R}^{3} \mid z \geq 0\right\}
$$

with buildings in it, represented by a polyhedron's body $B^{o}$

$$
B=\bigcup_{o=1}^{N_{o}} B^{o}
$$

where $N_{o}$ is the number of buildings. Then, a 3-D communication-feasible area for the possible position of the ground convoy $\mathbf{x}_{c v}=\left\{x_{c v}, y_{c v}, z_{c v}\right\} \in \mathbb{R}^{3}$ considering lineof-sight (LOS) block by buildings and the limited communication range $r_{c o m}^{\max }$ can be defined as:

$$
\mathbf{X}_{C F}\left(\mathbf{x}_{c v}\right)=\left\{(x, y, z) \in \mathbf{X}_{E} \mid \tilde{\mathbf{X}}_{c v} \cap B=\{\emptyset\}\right\}
$$

where

$$
\begin{aligned}
& \tilde{\mathbf{X}}_{c v}=\left\{(\tilde{x}, \tilde{y}, \tilde{z}) \in \mathbb{R}^{3} \mid \alpha \tilde{x}+\beta \tilde{y}+\gamma \tilde{z}\right. \\
& =\alpha\left(x_{c v}-x\right)+\beta\left(y_{c v}-y\right)+\gamma\left(z_{c v}-z\right)=0, \\
& \left.\left.\tilde{x}^{2}+\tilde{y}^{2}+\tilde{z}^{2} \leq\left(r_{c o m}^{\max }\right)^{2}\right)\right\} .
\end{aligned}
$$

Obtaining $\mathbf{X}_{C F}\left(\mathbf{x}_{c v}\right)$ exactly at an arbitrary convoy position $\mathbf{x}_{c v}$ where there are a large number of buildings in the environment would be computationally intractable. Thus, in practice, $\tilde{\mathbf{X}}_{c v}$ can be approximately computed by combining several discrete 2-D visibility polygons as used in [13], [14]. For a given convoy position, the 2-D cross section of visibility polygons with a limited communication range at a certain azimuth angle (e.g. East-Height cross section as shown in Fig. 2(a)) can be obtained while considering LOS block by corresponding buildings. Joining all cross section areas for 180 degrees sampled at a fixed angle interval results in the $3-\mathrm{D}$ communication-feasible area as shown in Fig. 2(b). Note that this sampling angle interval needs to be carefully determined considering the system requirement as it is a trade-off between the computation time, the data storage and the resolution of the communication-feasible area.

\section{B. Desired loitering orbit determination}

Once the communication-feasible area is generated, then UAVs should fly within the generated area in order to maintain the communication (and the LOS) to the convoy at all times. Although there might be several ways to do so, we adopt a loitering (or standoff) orbit tracking concept [15] [17] to this problem, which makes the UAV follow a loitering orbit determined by the communication-feasible area. This has several benefits over other approaches (e.g. just flying within the area [13] or following the exact area boundary): prevent sudden change of flight course and direction thus efficient under dynamic constraints of a fixed-wing UAV; path or behaviour is predictable to a certain extent as it loiters around a known point; straightforward collision avoidance between UAVs if multiple UAVs are involved by enforcing angular separation on the same orbit; orbiting around the 


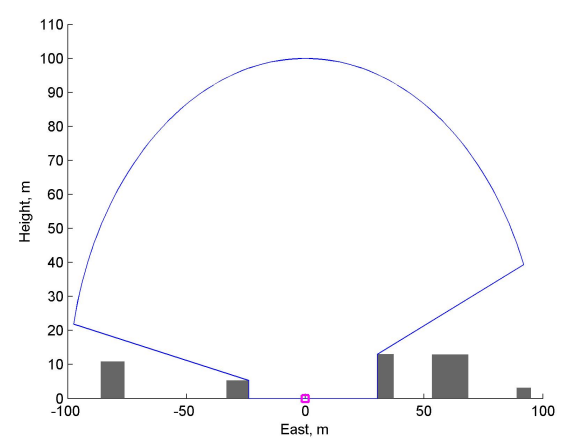

(a) 2-D

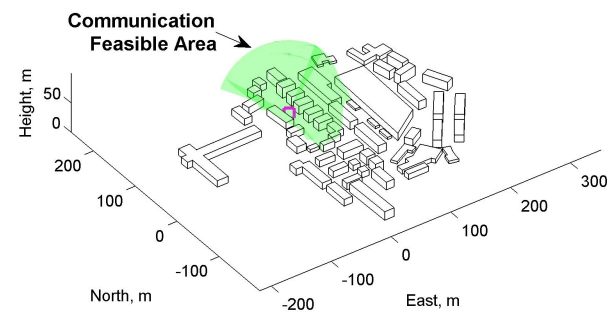

(b) 3-D

Fig. 2. Communication-feasible area generation.

ground vehicle would allow to gather more information; and being able to be as close as possible to the convoy all the time (hence, better communication quality and network connectivity) while satisfying the movement constraints.

Note that, if the UAV flies at a low altitude, then the communication-feasible area would not be enough for it to stay within due to the minimum turning radius, as illustrated in Fig. 3. On the other hand, at a high altitude, even though there would be enough space for the UAV to move around but the distance between the ground convoy and the UAV is large in this case. Consequently, finding the minimum height $h_{d}$ and a loitering centre $\bar{r}_{c t, d}$ in the generated communicationfeasible area in which the UAV can stay becomes a necessary task, which can be formulated as:

$$
\begin{array}{ll} 
& \left(\overline{\mathbf{r}}_{c t, d}, h_{d}\right)=\underset{\overline{\mathbf{r}}_{c t}, h}{\arg \min } h \\
\text { s.t. } & \left\|\overline{\mathbf{r}}_{c t}, \mathbf{B}_{p t, i}\left(\mathbf{X}_{C F}^{h}\right)\right\|_{2} \geq r_{t u r n}^{\text {min }}+\varepsilon=r_{d}, \\
& h \geq h_{\text {build }}^{\text {max }}, \quad \forall i \in\left\{1, \cdots, N_{p}\right\}
\end{array}
$$

where $\mathbf{B}_{p t, i}\left(\mathbf{X}_{C F}^{h}\right) \in\left\{\mathbf{B}_{p t, 1}, \cdots, \mathbf{B}_{p t, N_{p}}\right\} \subset \mathbb{R}^{2 \times N_{p}}$ represents the set of discretised boundary points of the communication-feasible area at a height of $h$ (i.e. $\mathbf{X}_{C F}^{h}=$ $\left.\left\{(x, y, z) \in \mathbf{X}_{C F} \mid z=h\right\}\right), N_{p}$ is the number of the boundary points and $\overline{\mathbf{r}}_{c t}=\sum_{i=1}^{N_{p}} \mathbf{B}_{p t, i}\left(\mathbf{X}_{C F}^{h}\right) / N_{p}$ is the mean position of them. $r_{\text {turn }}^{\text {min }}$ is the minimum turning radius of the UAV and $\epsilon$ is a positive margin, so $r_{d}=r_{\text {turn }}^{m i n}+\varepsilon$ is a desired loitering orbit radius. $h_{\text {build }}^{\max }$ represents the maximum height of the buildings.

\section{GP regression on desired loitering orbits}

The above communication-feasible area and the corresponding loitering orbit could be generated in real time

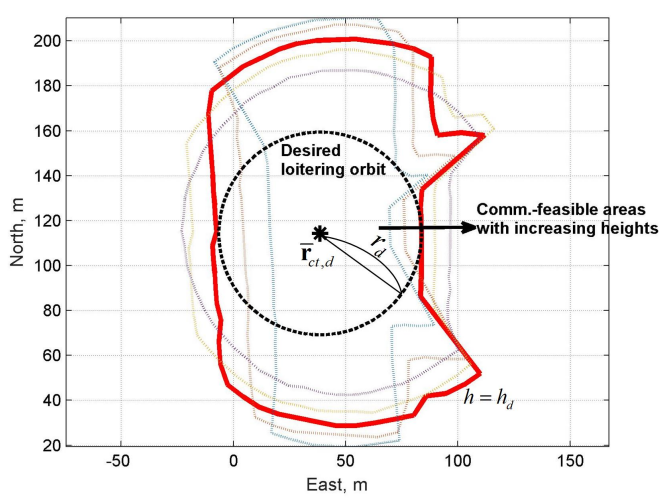

Fig. 3. Communication-feasible area cross section at $h=h_{d}$.

for each convoy position whenever needed. However, if the online computation capacity is limited, the part of this process can be made offline by using machine learning algorithms. Among others, the Gaussian Process regression (GPR) is used in this study [18]. The Gaussian Process (GP) can be viewed as a Gaussian distribution over functions, and it can be used to infer or predict function values at a finite set of test points using the observed data. Regression using the GP is briefly explained as follows [18].

Firstly, a standard regression model is defined as $y_{G P}=$ $f(\mathbf{x})+\epsilon$, where $\mathbf{x}$ is an input vector of dimension $d$, and $y_{G P}$ is a scalar output. The noise $\epsilon$ is assumed to be an independent and identically distributed Gaussian distribution with zero mean and variance $\sigma^{2}$. Then, the Gaussian Process $f(\mathbf{x})$ is specified by its mean function $m(\mathbf{x})=\mathbb{E}[f(\mathbf{x})]$ and the covariance function $k\left(\mathbf{x}, \mathbf{x}^{\prime}\right)=\mathbb{E}\left[(f(\mathbf{x})-m(\mathbf{x}))\left(f\left(\mathbf{x}^{\prime}\right)-\right.\right.$ $\left.m\left(\mathbf{x}^{\prime}\right)\right)$ ]. Since this study assumes zero-mean GP, the process can be expressed as $f(\mathbf{x}) \sim G P\left(0, k\left(\mathbf{x}, \mathbf{x}^{\prime}\right)\right)$. A training set with $N_{t}$ observation is expressed as $\mathcal{D}=\left\{\left(\mathbf{x}_{n}, y_{G P, n}\right) \mid n=\right.$ $\left.1, \cdots, N_{t}\right\}=\left\{\mathbf{X}, \mathbf{y}_{G P}\right\}$, and the following squared exponential covariance function is used:

$$
k\left(\mathbf{x}, \mathbf{x}^{\prime}\right)=\sigma_{f}^{2} \exp \left(-\frac{1}{2}\left(\mathbf{x}-\mathbf{x}^{\prime}\right)^{\top} \sum\left(\mathbf{x}-\mathbf{x}^{\prime}\right)\right)
$$

where $\sigma_{f}$ determines the magnitude, and $\sum=l^{-2} I$ is an isotropic covariance function.

Given the GP model and the covariance function above, the fitness of this model to the training set $\mathcal{D}$ can be evaluated using the marginal likelihood conditioned on the hyperparameters $\theta$ (i.e. the parameters of the covariance function):

$$
\begin{aligned}
& \log p\left(\mathbf{y}_{G P} \mid \mathbf{X}, \theta\right)= \\
& -\frac{1}{2} \mathbf{y}_{G P}^{\top} \mathbf{K}_{y}^{-1} \mathbf{y}_{G P}-\frac{1}{2} \log \left|\mathbf{K}_{y}\right|-\frac{N_{t}}{2} \log 2 \pi
\end{aligned}
$$

where $\mathbf{K}_{y}=\mathbf{K}+\sigma^{2} I$ and $\mathbf{K}=k(\mathbf{X}, \mathbf{X})$. The hyperparameters are optimised to provide good predictions using the partial derivatives of Eq. (7) with respect to the hyperparameters using a gradient-based optimiser. Note that these hyperparameters are fixed once they are optimised with the training set. 
Given the training set $\mathcal{D}$, the covariance function with the trained hyperparameters, and a test input vector $\mathbf{x}_{*}$, the predictive distribution for the GPR can be computed as:

$$
f_{*} \mid \mathbf{X}, \mathbf{y}_{G P}, \mathbf{x}_{*} \sim \mathcal{N}\left(\bar{f}_{*}, \mathbb{V}\left[f_{*}\right]\right)
$$

where the mean and variance are defined as:

$$
\begin{aligned}
\bar{f}_{*} & =\mathbf{k}_{*}^{\top}\left(\mathbf{K}+\sigma_{n}^{2} I\right)^{-1} \mathbf{y}_{G P} \\
\mathbb{V}\left[f_{*}\right] & =k\left(\mathbf{x}_{*}, \mathbf{x}_{*}\right)-\mathbf{k}_{*}^{\top}\left(\mathbf{K}+\sigma_{n}^{2} I\right)^{-1} \mathbf{k}_{*}
\end{aligned}
$$

where $\mathbf{k}_{*}$ denotes the vector of covariance between the test and the training points.

In this study, the centre of the desired loitering orbit $\left(\overline{\mathbf{r}}_{c t, d}\right)$ and its height $\left(h_{d}\right)$ at certain points $(4 \mathrm{~m}$ by $4 \mathrm{~m}$ grids) in the area of interest are used as a training data set. Figure 4 shows the example result of the GPR on the desired loitering orbit height using $1 \mathrm{~m}$ by $1 \mathrm{~m}$ grids over a certain area as test points, and it shows the higher height around narrow roads as expected. Note that once the GPR process (in particular, hyperparameter optimisation) is done offline, the predicted output $\bar{f}_{*}$ (i.e. the desired orbit centre and height) at the current convoy position can be obtained in real time using Eq. (9).

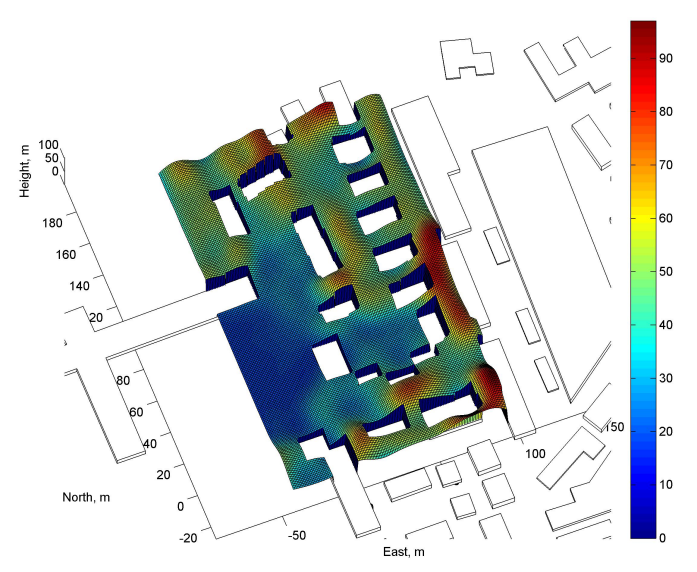

Fig. 4. The example result of the GPR on the desired height using 1 by $1 \mathrm{~m}$ grid test points over a certain area.

\section{LOITERING ORBit Following GUidAnCE}

This section presents a three-dimensional (3-D) dynamic model of the UAV and Lyapunov vector field guidance (LVFG)-based convoy following trajectory planning to follow the desire loitering orbit accurately. Note that the LVFG problem is formulated for a pair of UAVs to have redundancy in case that one UAV loses communication (i.e. going outside of the feasible area).

\section{A. 3-D UAV dynamic model}

Assuming each UAV has a low-level flight controller such as stability/controllability augmentation system for heading, flight path and velocity hold functions, this study aims to design guidance inputs to this low-level controller for standoff orbit tracking. Consider a 3-D UAV kinematic model by extending a 2-D model given in [16]:

$$
\left(\begin{array}{c}
\dot{x} \\
\dot{y} \\
\dot{h} \\
\dot{\chi} \\
\dot{\gamma} \\
\dot{v} \\
\dot{\omega}_{\chi} \\
\dot{\omega}_{\gamma}
\end{array}\right)=f(\mathbf{x}, \mathbf{u})=\left(\begin{array}{c}
v \cos \chi \cos \gamma \\
v \sin \chi \cos \gamma \\
v \sin \gamma \\
\omega_{\chi} \\
\omega_{\gamma} \\
-\frac{1}{\tau_{v}} v+\frac{1}{\tau_{\nu}} u_{v} \\
-\frac{1}{\tau_{\omega_{\chi}}} \omega_{\chi}+\frac{1}{\tau_{\omega_{\chi}}} u_{\omega_{\chi}} \\
-\frac{1}{\tau_{\omega_{\gamma}}} \omega_{\gamma}+\frac{1}{\tau_{\omega_{\gamma}}} u_{\omega_{\gamma}}
\end{array}\right)
$$

where $\mathbf{x}=\left(x, y, h, \chi, \gamma, v, \omega_{\chi}, \omega_{\gamma}\right)^{T}$ are the inertial 3-D position, heading (or course) angle, flight path angle, speed, and yaw and pitch rate of the UAV, respectively. $\tau_{v}, \tau_{\omega_{\chi}}$, and $\tau_{\omega_{\gamma}}$ are time constants for considering an actuator delay. $\mathbf{u}=\left(u_{v}, u_{\omega_{\chi}}, u_{\omega_{\gamma}}\right)^{T}$ are the commanded speed, turning rate and pitch rate constrained by the following limits: $\left|u_{v}-v_{0}\right| \leq$ $\Delta v_{\max },\left|u_{\omega_{\chi}}\right| \leq \omega_{\chi, \max }$, and $\left|u_{\omega_{\gamma}}\right| \leq \omega_{\gamma, \max }$ where $v_{0}$ is a nominal speed of the UAV. The continuous UAV model in (11) can be discretised by Euler integration into:

$$
\mathbf{x}_{k+1}=f_{d}\left(\mathbf{x}_{k}, \mathbf{u}_{k}\right)=\mathbf{x}_{k}+T_{s} f\left(\mathbf{x}_{k}, \mathbf{u}_{k}\right)
$$

where $\mathbf{x}_{k}=\left(x_{k}, y_{k}, h_{k}, \chi_{k}, \gamma_{k}, v_{k}, \omega_{\chi k}, \omega_{\gamma k}\right)^{T}, \mathbf{u}_{k}=$ $\left(u_{v k}, u_{\omega_{\chi} k}, u_{\omega_{\gamma} k}\right)^{T}$, and $T_{s}$ is a sampling time.

\section{B. Lyapunov Vector Field Guidance (LVFG)}

The LVFG uses the following vector field function to compute the desired velocity which makes the UAV orbit around the centre of the desired loitering orbit [15], [17]: $V_{l}(x, y)=\left(r^{2}-r_{d}^{2}\right)^{2}$ (Hereafter the subscript $k$ will be omitted for simple notation). Then, the total time derivative of $V_{l}$ is given by $\dot{V}_{l}=\nabla V_{l} \cdot[\dot{x}, \dot{y}]^{T}$, and the following desired velocity $\left[\dot{x}_{d}, \dot{y}_{d}\right]^{T}$ can make $\dot{V}_{l}$ nonpositive, which provides stable convergence to the orbit as shown in Fig. 5.

$$
\left[\begin{array}{c}
\dot{x}_{d} \\
\dot{y}_{d}
\end{array}\right] \frac{-v_{d}}{r\left(r^{2}+r_{d}^{2}\right)}\left[\begin{array}{c}
\delta x\left(r^{2}-r_{d}^{2}\right)+\delta y\left(2 r r_{d}\right) \\
\delta y\left(r^{2}-r_{d}^{2}\right)-\delta x\left(2 r r_{d}\right)
\end{array}\right]
$$

where $\delta x=x-\bar{x}_{c t, d}, \delta y=y-\bar{y}_{c t, d}, r=\sqrt{\delta x^{2}+\delta y^{2}}$ is the distance between the UAV and the loitering orbit centre, Herein $\overline{\mathbf{r}}_{c t, d}=\left(\bar{x}_{c t, d}, \bar{y}_{c t, d}\right)^{T}$ is the loitering orbit centre obtained as described in Eq. (5). $v_{d}$ is a desired UAV speed. The guidance command $u_{\omega_{\chi}}$ for the UAV turn rate is computed by proportional feedback and feedforward control terms as:

$$
u_{\omega_{\chi}}=-k_{\omega_{\chi}}\left(\psi-\psi_{d}\right)+\dot{\psi}_{d}
$$

where the desired heading $\psi_{d}$ can be determined using the desired velocity in Eq. (13) as: $\psi_{d}=\tan ^{-1}\left(\dot{y}_{d} / \dot{x}_{d}\right)$ and differentiating $\psi_{d}$ gives $\dot{\psi}_{d}$. Note that for the guidance command to be feasible (i.e. within $\omega_{\max }$ ), the control gain $k_{\omega}$ needs to be carefully determined. Height control is done by a simple feedback control, given by:

$$
u_{\omega_{\gamma}}=-k_{\omega_{\gamma}}\left(h-h_{d}\right)
$$

where $h_{d}$ is a desired height for UAVs to maintain. 


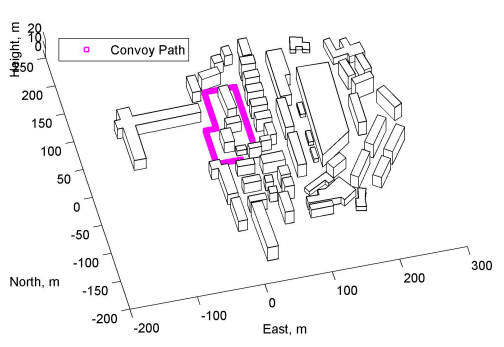

(a) Case 1 (70 seconds)

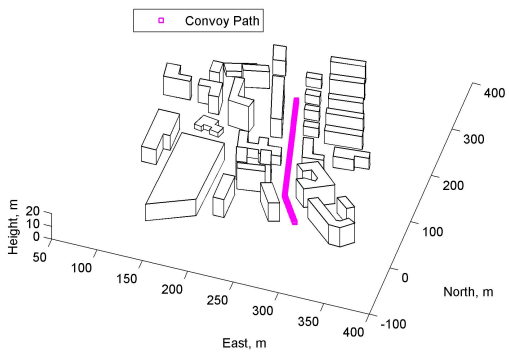

(b) Case 2 (45 seconds)

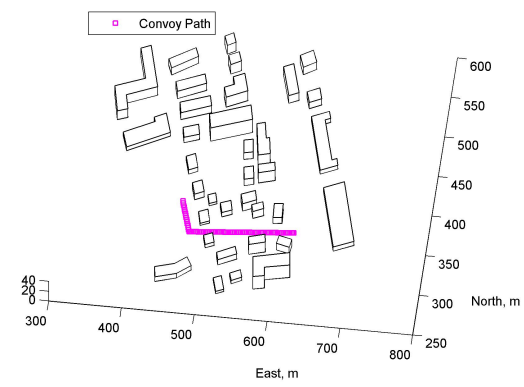

(c) Case 3 (40 seconds)

Fig. 6. Sample convoy following scenarios.

TABLE I

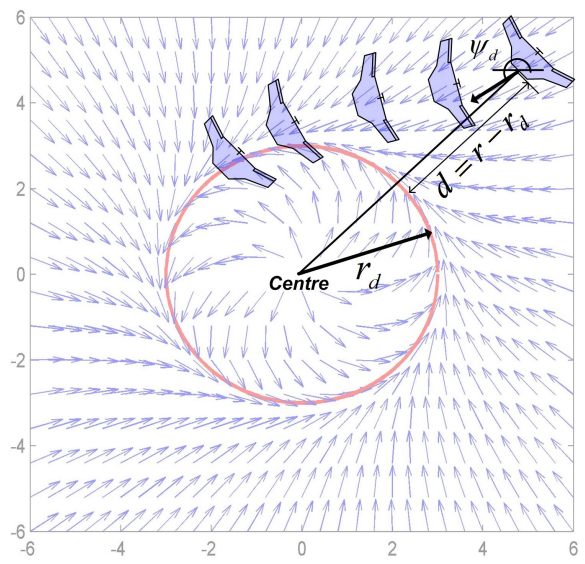

Fig. 5. Illustration of vector field and loitering orbit following.

If multiple UAVs follows the desired loitering orbit while maintaining a certain angular separation between them, it is more likely for at least one UAV to be in the communicationfeasible area. To achieve this, speed of each UAV is controlled as given [15]:

$$
u_{v}= \pm k_{v}\left(\Delta \theta-\theta_{d}\right) r_{d}+v_{d}
$$

where $k_{v}$ is a control gain, $\Delta \theta$ is the angular phase separation between UAVs, and $\theta_{d}$ is a desired phase difference between the UAVs.

\section{NUmerical Simulations}

This section carries out numerical simulations using the proposed convoy following trajectory planning for a moving ground convoy. A simulation sampling time $T_{s}$ is set to $0.5 \mathrm{~s}$ and the parameter setting for the simulation is shown in Table I. Three sample scenarios are considered where the convoy moves through the urban area surrounded by a number of buildings as shown in Fig. 6 .

A sample capture of the communication-aware convoy following simulation result is shown in Fig. 7. Two UAVs follow the same desired orbit at the desired height, and as a result they are within the communication-feasible area, which ensures the communication to the ground convoy.
SIMULATION PARAMETERS

\begin{tabular}{llc}
\hline Parameter & Value & Unit \\
\hline \hline$\theta_{d}$ & $\pi$ & $\mathrm{rad}$ \\
$v_{0}, v_{d}$ & 20 & $\mathrm{~m} / \mathrm{s}$ \\
$r_{d}$ & 45 & $\mathrm{~m}$ \\
$\Delta v_{\max }$ & 10 & $\mathrm{~m} / \mathrm{s}$ \\
$\left(\omega_{\chi}, \max , \omega_{\gamma, \max }\right)$ & $(0.6,0.15)$ & $\mathrm{rad} / \mathrm{s}$ \\
$\tau_{v}, \tau_{\omega_{\chi}}, \tau_{\omega_{\gamma}}$ & $1 / 3$ & $\mathrm{sec}$ \\
$\left(k_{v}, k_{\omega_{\chi}}, k_{\omega_{\gamma}}\right)$ & $(0.12,2,0.5)$ & - \\
\hline
\end{tabular}

Table II shows the performance of the LVFG in terms of orbit distance (i.e. $\left|r-r_{d}\right|$ ), height, and angular separation. The number of instances when the UAV is outside of the communication-feasible area is also included. By using two UAVs, those instances (i.e. out-of-communication for both UAVs at the same time) are reduced significantly.

TABLE II

TRACKING PERFORMANCE FOR SAMPLE SCENARIOS

\begin{tabular}{lccc}
\hline Mean error & Case 1 & Case 2 & Case 3 \\
\hline \hline Orbit distance $(\mathrm{m})$ & 10.51 & 10.72 & 13.10 \\
Height $(\mathrm{m})$ & 5.80 & 6.77 & 5.41 \\
Angular separation $(\mathrm{deg})$ & 23.05 & 31.36 & 24.22 \\
Out of Comm.* & $28 / 27(5)$ & $26 / 14(9)$ & $14 / 16(1)$ \\
\hline *UAV1/UAV2 (out of comm. for both UAVs at the same time)
\end{tabular}

Figure 8 shows the capture of the indoor flight test ${ }^{1}$. Due to the indoor space limitation, we used the quadrotor UAV which attempts to stay at the centre the desired loitering orbit (rather than loitering around it). Following the centre of the desired orbit is still meaningful to make the trajectory planning algorithm robust to the navigational position error or position control error since as long as the quadrotor is within the loitering orbit, communication to the convoy is expected to be ensured.

\section{Vi. CONClusions And Future work}

This paper proposed a communication-aware trajectory planning approach which makes the UAV stay within the

\footnotetext{
${ }^{1} \mathrm{~A}$ short video including the indoor flight test and some numerical simulation results is provided at the following link:

http: \\dl.dropboxusercontent.com/u/17047357/COMM_MED.zip.
} 


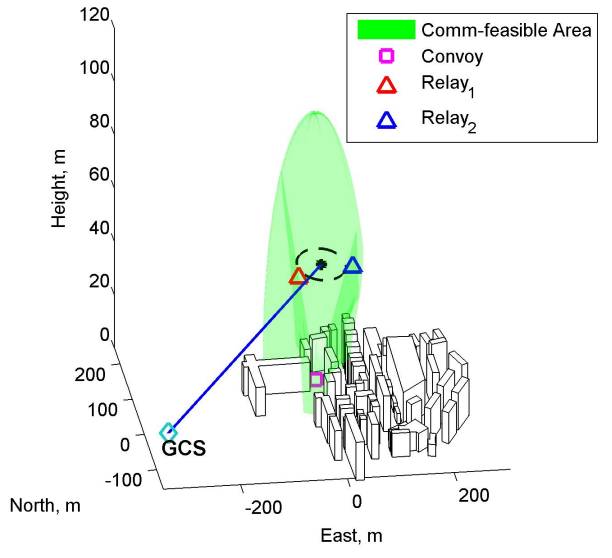

(a) Top-down view

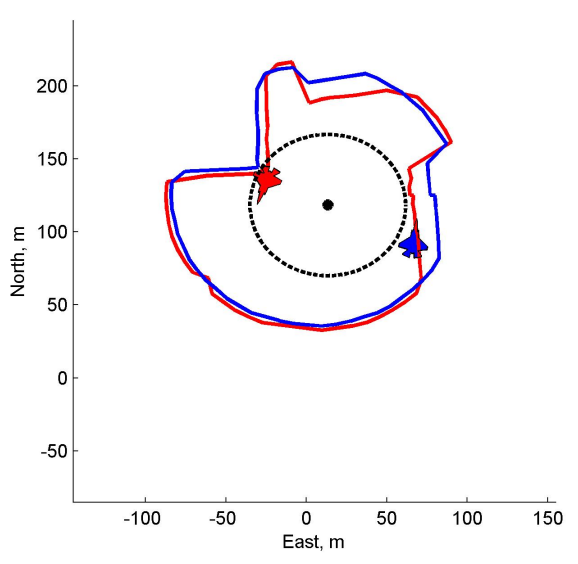

(b) Cross-section view

Fig. 7. A sample capture of communication-aware convoy following simulation for the Case 1 scenario with different views.

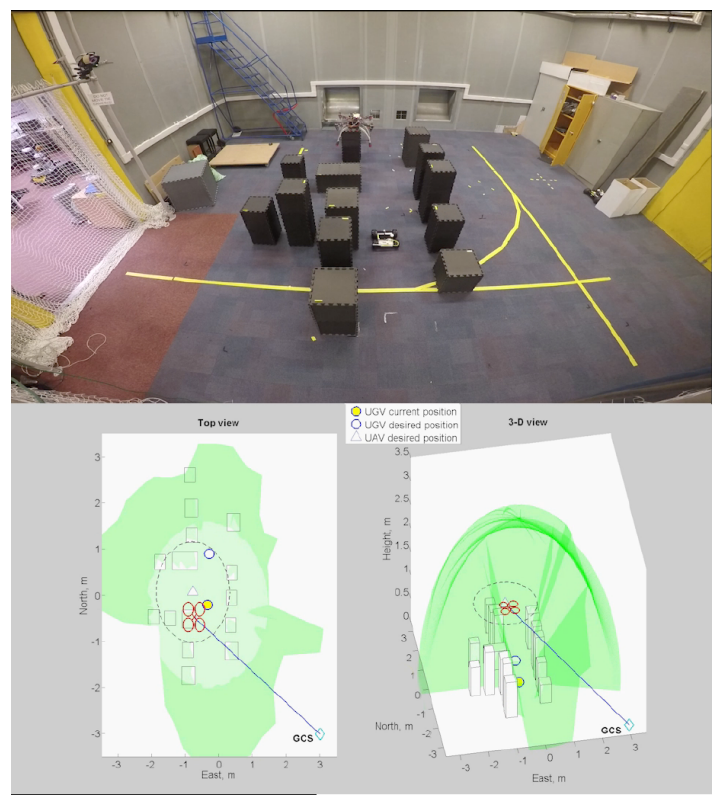

Fig. 8. Captured image from the indoor flight test for communication-aware convoy following. communication-feasible area against a moving ground convoy in an urban environment. Numerical simulations and a proof-of-concept flight test showed the feasibility of the approach. To improve the loitering orbit following performance and thus achieving better communication (i.e. fewer out-ofcommunication instances), prediction-based approaches (e.g. nonlinear model predictive control [16]) will be applied by utilising future information on the communication-feasible area computation. Robust planning under uncertainty on a convoy position will also be followed as future work.

\section{REFERENCES}

[1] H.S. Shin and P. Segui-Gasco. UAV Swarms: Decision-Making Paradigms. Encyclopedia of Aerospace Engineering. Wiley \& Sons, Ltd., 2014.

[2] P. Basu, J. Redi, and V. Shurbanov. Coordinated flocking of UAVs for improved connectivity of mobile ground nodes. In IEEE Military Communications Conference (MILCOM 2004), 2004.

[3] S. Kim, P. Silson, A. Tsourdos, and M. Shanmugavel. Dubins path planning of multiple unmanned airborne vehicles for communication relay. Proceedings of the Institution of Mechanical Engineers, Part G: Journal of Aerospace Engineering, 225(1):12-25, 2011.

[4] S.S. Ponda, L.B. Johnson, A.N. Kopeikin, H.L. Choi, and J.P. How. Distributed planning strategies to ensure network connectivity for dynamic heterogeneous teams. IEEE Journal on Selected Areas in Communications, 30(5):861-869, 2012.

[5] J.J. Acecedo, C. Begona, I. Maza, and A. Ollero. Distributed approach for coverage and patrolling missions with a team of heterogeneous aerial robots under communication constraints. International Journal of Advanced Robotic Systems, 10(28):1-13, 2013.

[6] K.J. Obermeyer, A. Ganguli, and F. Bullo. Multi-agent deployment for visibility coverage in polygonal environments with holes. International Journal on Robust and Nonlinear Control, 21(12):1467-1492, 2011.

[7] H. Zhu, A.L. Swindlehurst, and K. Liu. Optimization of MANET connectivity via smart deployment/movement of unmanned air vehicles. IEEE Transactions on Vehicular Technology, 58(7):3533-3546, 2009.

[8] S. Kim, H. Oh, J. Suk, and A. Tsourdos. Coordinated trajectory planning for efficient communication rely using multiple UAVs. Control Engineering Practice, 29:42-49, 2014.

[9] S. Hauert, L. Winkler, J.C. Zufferey, and D. Floreano. Ant-based swarming with positionless micro air vehicles for communication relay. Swarm Intelligence, 2:167-188, 2008.

[10] E.W. Frew and T.X. Brown. Airborne communication networks for small unmanned aircraft systems. Proceedings of the IEEE, 96(12):2008-2027, 2008

[11] J. Le Ny, A. Ribeiro, and G.J. Papas. Adaptive communicationconstrained deployment of unmanned vehicle systems. IEEE Journal on Selected Areas in Communications, 30(5):923-934, 2012.

[12] J. Kim and Y. Kim. Moving ground target tracking on dense obstacle area using UAVs. In 17th IFAC World Congress, Seoul, Korea, 2008.

[13] V. Shaferman and T. Shima. Unmanned aerial vehicles cooperative tracking of moving ground target in urban environments. Journal of Guidance, Control and Dynamics, 31(5), 2008.

[14] R.G. Vilar and H.S. Shin. Communication-aware task assignment for uav cooperation in urban environments. In IFAC Workshop on Research, Development and Education on Unmanned Aeral Systems, Compiegne, France, November 2013.

[15] E.W. Frew, D.A. Lawrence, and S. Morris. Coordinated standoff tracking of moving targets using Lyapunov guidance vector fields. Journal of Guidance, Control, and Dynamics, 31(2), 2008.

[16] S. Kim, H. Oh, and A. Tsourdos. Nonlinear model predictive coordinated standoff tracking of moving ground vehicle. Journal of Guidance, Control and Dynamics, 36(2):557-566, 2013.

[17] H. Oh, S. Kim, H. Shin, and A. Tsourdos. Coordinated standoff tracking of moving target groups using multiple UAVs. IEEE Transactions on Aerospace and Electronic Systems, 51(2):1501-1514, 2015.

[18] C.E. Rasmussen and C.K.I. Williams. Gaussian Process for Machine Learning. MIT Press, 2006. 